\title{
Dimensiones del Empoderamiento Digital y Currículo para el Sector Universitario
}

\author{
Iris A. Jiménez ${ }^{(1)^{*}}$, Raúl J. Martelo ${ }^{(2)}$ y José D.C. Jaimes ${ }^{(3)}$ \\ (1) Universidad de la Guajira, Facultad de Ciencias Básicas y Aplicadas, Grupo de Investigación BIEMARC, \\ Km 5 vía a Maicao, Guajira-Colombia. (e-mail: irisjmenezpitre12@gmail.com) \\ (2) Universidad de Cartagena, Facultad de Ingeniería, Grupo de Investigación en Tecnologías de las \\ Comunicaciones e Informática (GIMATICA), Avenida el Consulado, Calle 30 No. 48-152. Cartagena, Bolívar- \\ Colombia. \\ (3) Universidad de Cartagena, Facultad de Enfermería, Grupo de Investigación en Medio Ambiente, \\ alimentos y Salud (MAAS), Cra. 50 \#24-120, Cartagena, Bolívar.
}

* Autor a quien debe ser dirigida la correspondencia

Recibido Oct. 7, 2016; Aceptado Dic. 23, 2016; Versión final Ene. 20, 2017, Publicado Ago. 2017

\section{Resumen}

Se analizan dos de las dimensiones del empoderamiento digital, relacionadas con la equidad digital y la empleabilidad dentro del sector universitario colombiano, específicamente en la Universidad de La Guajira. Bajo una metodología descriptiva, transeccional y con un diseño de investigación que se corresponde con un estudio de caso, se diseñó un instrumento de recolección de datos, aplicado a una muestra de 125 docentes. Los resultados demuestran un claro rezago en las posibilidades de acceso a las Tecnologías de Información y Comunicación en condiciones de equidad que sin duda afectaran los niveles de empleabilidad de los futuros trabajadores profesionales. De allí que en las conclusiones se propongan reorientaciones curriculares para este sector con miras a superar las dificultades detectadas. Las conclusiones también destacan que se debe seguir avanzando en la capacitación docente para el uso pedagógico de estas tecnologías y ofrecer apoyo a los estudiantes para su verdadero empoderamiento digital.

Palabras clave: empoderamiento digital; equidad digital; empleabilidad; tecnologías de información; currículo.

\section{Dimensions of Digital Empowerment and Curriculum for the Higher Education Sector}

\begin{abstract}
In this article two dimensions of digital empowerment related to digital equity and employability are analyzed within the Colombian higher education sector, specifically at the University of La Guajira. Under a descriptive and transactional methodology and employing a case study research design, a data collection instrument was made and applied to a sample of 125 teachers. The results show a clear backwardness in the possibilities for accessing technologies of information and communication in equity conditions, which certainly will affect employability levels of the future professional workers. As a consequence, conclusions regarding curriculum reorientations are proposed for this sector for overcoming the identified difficulties. The conclusions also highlight that teachers' training for the pedagogical use of these technologies must continue and that students must receive appropriate support for their real digital empowerment.
\end{abstract}

Keywords: digital empowerment; digital equity; employment; technologies of information; curriculum 


\section{INTRODUCCIÓN}

En la actualidad, el auge experimentado por las políticas de globalización y la introducción de herramientas derivadas de la Tecnología de Información y Comunicación (TIC), como medios preponderantes para la generación de la sociedad de la información y el conocimiento, han impuesto retos que se traducen en nuevos procedimientos de desarrollo económico, educativo, social y cultural. Dentro de este escenario, la educación, especialmente la universitaria, se erige como camino certero al convertirse en una vía estratégica capaz de favorecer el desarrollo de estos procesos, fortalecer la cohesión social, los valores de la vida democrática y su institucionalidad e irrumpir sobre las estructuras y las dinámicas de exclusión sociocultural (Bauman, 2005). En este sentido, el verdadero desafío de la innovación educativa basada en las ventajas y las potencialidades de las TIC consiste en pensar a priori cuáles son los objetivos más trascendentales de la educación situada en un tiempo y espacio determinado, además de dirimir en las formas y las condiciones de su incorporación. Se trata de tomar en cuenta lo que estas tecnologías pueden aportar al diseño de una oferta educativa de calidad en la cual se demuestre el compromiso de superación de las prácticas de segregación y negación sociocultural (Tedesco, 2005). Tal desafío, trasladado al contexto universitario no sólo implica una educación con sentido de equidad e igualdad, sino que también demanda la formación de individuos empoderados digitalmente para que puedan desarrollar exitosamente competencias de empleabilidad e insertarse, sin ninguna dificultad, en el mercado productivo de este mundo globalizado.

La educación universitaria igualitaria, equitativa y destinada al empoderamiento digital de la comunidad requiere de una transformación curricular que vaya desde el diseño didáctico hasta la práctica cotidiana de aula. Así lo sugiere De La Fontaine y Aparicio (2008) cuando afirman que no basta con agregar dispositivos tecnológicos, sino integrar la concepción y estrategias intrínsecamente unidas a metodologías de inclusión TIC que desarrollan y promueven procesos de mejora de las condiciones de vida, comunicación, participación y poder de los grupos menos atendidos. En lo que respecta al empoderamiento, como uno de los tres conceptos centrales de este trabajo, Gisbert y Esteve (2011) coinciden en definirlo como el conjunto de competencias digitales que poseen los individuos y que los convierte en seres más eficientes, críticos y con unas habilidades personales y profesionales que van más allá del simple uso de la tecnología, ya que la misma le permite buscar, captar, gestionar y tratar la información, presentar y difundir los contenidos en el formato adecuado y comunicarse y colaborar en la red.

Asimismo, Gisbert (2011) opina que estas competencias digitales son la suma de las habilidades, conocimientos y actitudes, en aspectos tecnológicos, informacionales, multimedia y comunicativos, dando lugar a una compleja alfabetización múltiple. Visión ésta que crea una nueva panorámica en cuanto a cómo identificar las competencias digitales de un estudiante universitario y que debe ser asumida por el docente de hoy en día dentro del contexto de la sociedad de la información y del conocimiento.

Por otra parte, Granados-Romero et al. (2014), en su estudio sobre las tecnologías de la información y las comunicaciones (TIC), las del aprendizaje y del conocimiento (TAC) y las tecnologías para el empoderamiento y la participación (TEP), como instrumentos de apoyo al docente de la universidad del siglo XXI, destacan que las TEP son aquellas tecnologías aplicadas para fomentar la participación de los "ciudadanos" en temas de índole político o social generando, de esta forma, una especie de empoderamiento y concientización de su posición en la sociedad, que se traduce en expresiones de protesta y/o acción pública. De allí que, tal como lo aseveran Catts y Lau (2008) en un informe de la UNESCO: "Hoy en día, los docentes en ejercicio necesitan estar preparados para ofrecer a sus estudiantes oportunidades de aprendizaje apoyadas en las TIC; para utilizarlas y para saber cómo estas pueden contribuir al aprendizaje de los estudiantes. Capacidades estas que actualmente forman parte integral del catálogo de competencias profesionales básicas de un docente". Esta aseveración de la UNESCO evidencia la preocupación de esta importante institución en la formación del docente y particularmente sobre el uso que le pueden dar éstos a las TIC para enriquecer el aprendizaje de los estudiantes.

En términos generales, el empoderamiento digital debe ser considerado como un proceso multidimensional de carácter social en donde el liderazgo, la comunicación y los grupos autodirigidos reemplazan la estructura piramidal mecanicista por una estructura más horizontal. Dentro de estas dimensiones se encuentran la accesibilidad, la cual debe ser provista por el Gobierno a través de una red de banda ancha para que todos los individuos tengan la oportunidad de hacer uso de las potencialidades que ofrecen las TIC de manera equitativa, evitando así las brechas digitales que han traído como consecuencia desigualdades en lo que concierne a la inclusión social (Berrío-Zapata y Rojas, 2014).). Como ejemplo de estas desigualdades se observan individuos que egresan de las universidades plenamente formados en el uso de las TIC para competir en el ámbito laboral, mientras que otros carecen de habilidades digitales para formar parte de esta competencia. Parte de este proceso multidimensional que involucra estar empoderado digitalmente se muestra en la figura 1, a continuación. 


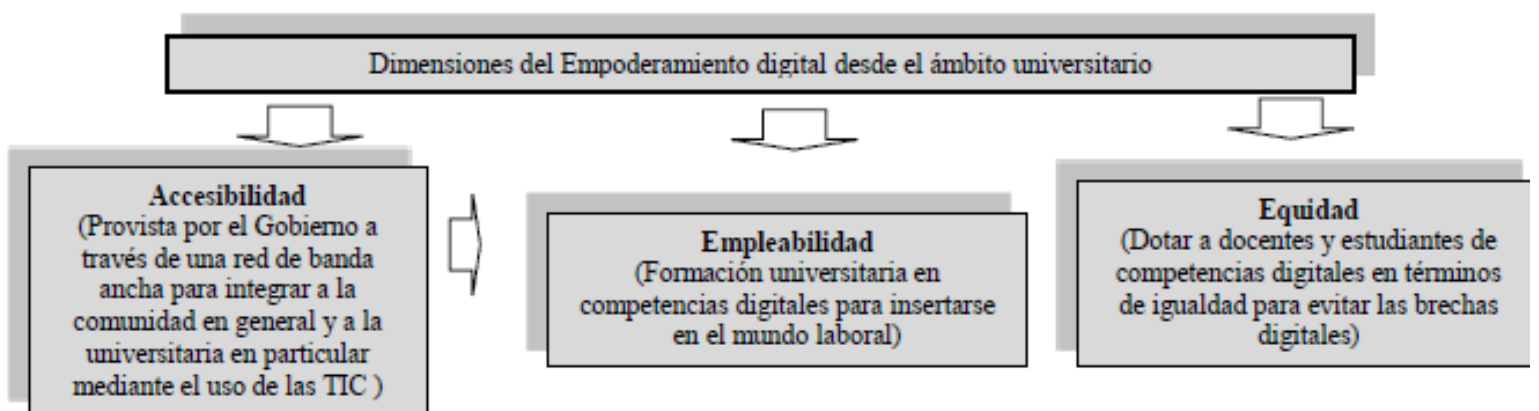

Fig. 1: Dimensiones del empoderamiento digital desde el ámbito universitario

La figura antes expuesta resume de manera sucinta las ventajas y características de un individuo empoderado digitalmente mediante el conocimiento que debe proveerle el sector universitario en materia de competencias digitales. Sin embargo, de acuerdo con las últimas investigaciones realizadas por expertos en el área y reportes emanados por organismos internacionales (WEB Foundation, 2015; Galvis, 2014; OEI, 2010), aún persisten patrones tradicionales que hacen caso omiso de las potencialidades que ofrecen las TIC como soportes en los procesos de aprendizaje y como herramientas que debe dominar el profesional en formación para empoderarse digitalmente y lograr un mejor desempeño en el mercado laboral.

Al tratar la equidad e igualdad, como otro de los tópicos centrales de esta investigación y en función de la dotación de habilidades digitales tanto a profesores como a estudiantes dentro del contexto universitario, se hace perentorio citar la opinión de Venkatesh y Sykes (2013) quienes establecen que las diferencias socioculturales y de contexto entre comunidades desarrolladas y excluidas son significativas, por tanto no puede ser asumido que los elementos críticos de apropiación de las TIC sean los mismos. En este orden de ideas, Berrío-Zapata (2005) exploró el problema de la medición de los efectos de las TIC en un grupo de estudiantes de pregrado en tres universidades privadas en Bogotá. Este estudio reportó que los estudiantes tienen acceso a las condiciones instrumentales asociadas con la utilización de las TIC pero no desarrollan su utilidad productiva de manera equitativa, dado que en su entorno no se valora la gestión de conocimiento e innovación ni se la premia social o económicamente.

En lo que respecta a las competencias digitales por parte de la población de docentes, se cita la investigación realizada por Quintero et al. (2011) con una muestra de noventa y cinco (95) profesores de planta, veinte y nueve (29) profesores ocasionales, trescientos noventa y cuatro (394) profesores catedráticos, los cuales suman un total de quinientos dieciocho (518) sujetos pertenecientes a la Universidad de La Guajira. Los hallazgos demuestran que no existe una política de integración organizada, sistemática y planeada por parte de la universidad, pues no todos los docentes usan las TIC, y los que las usan lo hacen de forma técnica y superficial como instrumento de trabajo, fuente informativa, pero sin un sólido planteamiento didáctico y sin una integración en el currículo y programación de aula. Tales hallazgos motivaron el desarrollo de la presente investigación constituyéndose en el problema a corroborar a lo largo de la misma.

En torno a la equidad digital, Tedesco (2005) esboza un panorama sobre las principales dificultades que presentaba Latinoamérica al momento de la implementación de las TIC a partir de la década de los 90. Entre éstas se encuentran: la segregación escolar de los grupos desaventajados y vulnerables; el desfase tecnológico, metodológico y didáctico imperante en las instituciones de formación pública; la deserción y la repitencia escolar; el disgregamiento de los contenidos curriculares; la inadecuada formación de los profesores y la pauperización de las condiciones laborales del personal docente, técnico y directivo; la dispersión de las inversiones financieras en el campo educativo; el hermetismo y autoreferencialidad curricular de la oferta educativa sin correspondencia con las demandas del contexto local y la vigencia de modelos pedagógicos caducos y arcaicos.

En cuanto a la empleabilidad, corresponde al sector universitario empoderar digitalmente al individuo en formación para que sea capaz de insertarse en el mundo laboral (Martínez et al., 2015). Para ello, se debe tomar en cuenta que, dentro de su curriculum, términos como la globalización, la sociedad del conocimiento, las TIC y las competencias profesionales deben conjugarse en un concierto mundial, liderado por la UNESCO, cuya tendencia es eliminar las brechas tecnológicas que puedan llegar a discriminar algunos sectores en este mundo permeado por las TIC (Bustos y Coll, 2010). En este sentido, el estudiante debe acreditar el desarrollo de competencias que son potencialmente útiles en las actividades profesionales y a la sociedad en general (Coll y Zegwaard, 2006) las cuales le permiten enlazar su formación con las necesidades del mercado laboral. 
Sobre la base de lo antes expuesto, es necesario aclarar que aun cuando el compromiso de empoderar digitalmente a los ciudadanos no constituye una responsabilidad exclusiva de las universidades, se está de acuerdo con lo planteado por Belloso y Perozo (2009) en relación con el empleo. De acuerdo con estos autores, el empleo como necesidad fundamental de toda sociedad, puede estar represado en los viejos paradigmas, sin permitir que tanto la oferta como la demanda estén en claros escenarios digitales. De allí que las organizaciones, grupos profesionales y especialmente las universidades puedan estar en conectividad para conocer sobre las disponibilidades así como de las oportunidades relacionadas al empleo, permitiendo una interacción casi en tiempo real para la ubicación de una plaza o la propuesta de una alternativa.

Con respecto a la pertinencia en la preparación universitaria y el vínculo con el mercado laboral, el Ministerio de las Tecnologías y Comunicaciones (MinTIC, 2008) a objeto de obtener este tipo de información creó desde el 2004, el Observatorio Laboral de la Educación. Este sistema informa sobre la vinculación de egresados al mercado laboral, la oferta de empleo para las distintas profesiones, los salarios y otras variables que inciden en la relación educación-trabajo. En este mismo orden, MinTIC (2008), también creó el Sistema de Prevención de la Deserción en Instituciones de Educación Superior (SPADIES) con el fin de combatir la deserción en educación superior. Dicho sistema ha permitido que un número importante de instituciones adelanten programas con base en el análisis de la información de sus estudiantes y de los factores que los ponen en riesgo, con el propósito de favorecer a su retención. En la práctica, se ha constituido un sistema de información que integra las necesidades de la educación superior (MinTIC, 2008).

Dentro del contexto colombiano, existen argumentos y opiniones encontradas que reportan fortalezas y debilidades de los logros alcanzados por parte del sector universitario con respecto a la equidad digital y la empleabilidad como dos de las dimensiones del empoderamiento digital. Las fortalezas se evidencian a través los progresos que ha hecho Colombia desde la época de los 90, en relación con la implementación de las Tecnologías de Información y Comunicación para la Educación (TICE), a los que se les ha otorgado referentes legales para garantizar su ejecución. Galvis (2014) resume tales referentes en 4 repertorios reglamentarios los cuales se ilustran en la Tabla 1.

Tabla 1: Basamentos legales colombianos sobre la implementación de las TIC.

\begin{tabular}{|l|l|}
\hline \multicolumn{1}{|c|}{ Basamentos legales } & \multicolumn{1}{c|}{ Propósito } \\
\hline Los planes de gobierno & $\begin{array}{l}\text { Cada cuatro años, orientan el desarrollo nacional y sectorial de la educación, la } \\
\text { mayoría de los cuales incluyen planes sobre TICE. }\end{array}$ \\
\hline $\begin{array}{l}\text { Los planes decenales } \\
\text { de Educación y de TIC }\end{array}$ & $\begin{array}{l}\text { Recogen propuestas de los distintos estamentos y se constituyen en la carta de } \\
\text { navegación más allá de las propuestas del gobierno de turno. }\end{array}$ \\
\hline Otros referentes & $\begin{array}{l}\text { Estudios de las misiones de sabios, los documentos CONPES emanados del } \\
\text { Departamento Nacional de Planeación (DNP) y la Visión Colombia 2019. }\end{array}$ \\
\hline Lineamientos & $\begin{array}{l}\text { Emitidos por DNP, MinEducación o MinTIC, acerca de elementos clave para hacer } \\
\text { operativa la política de TICE. }\end{array}$ \\
\hline
\end{tabular}

Sobre la base de esta fundamentación legal, ha sido notorio el rol de las universidades colombianas en cuanto a la preocupación de dotar a la comunidad académica de habilidades digitales. Como líderes de la innovación, apoyadas en las TICE, estas Instituciones de Educación Superior (IES) han desarrollado proyectos, bien en colaboración con el Ministerio de Educación Nacional (MEN) o con otras firmas especializadas. Entre estos proyectos, reportados por Galvis (2014), se encuentran: Conexiones, Ludomática, Pequeños Científicos, Matemáticas y TIC, Escuela Virtual, Temáticas, Maestro Digital, Formación docente en TIC, AQTCR, Transformando la Práctica Docente, por nombrar algunos.

En relación con las debilidades de la educación superior para dotar a su comunidad académica de habilidades digitales, Mejía y López (2016) describen serias dificultades para el desarrollo tecnológico en las distintas funciones de las IES a través de las TIC, manifestando que procesos como por ejemplo los del elearning en las instituciones de educación superior colombianas se encuentran desarticulados de los procesos misionales, debido a que en su gran mayoría, estos nacen de iniciativas particulares y nunca como producto de una reflexión de la filosofía institucional y la revisión de los lineamientos estratégicos, planes de desarrollo y programas en el largo plazo. De igual manera, estos autores afirman que el nivel de empoderamiento en las instancias directivas sobre este tipo de proyectos y los procesos de comunicación de la visión en muy pocos casos han estado estructurados y debidamente realizados. Este se debe a que casi todas las universidades estudiadas, el uso de las redes sociales es deficiente y, además, carecen de indicadores que den cuenta del mejoramiento permanente de los lineamientos académicos para el uso de las TIC. Por tal motivo, no se han introducido como medios de aprendizaje y comunicación, pues hoy son las que marcan tendencia en los sistemas de interacción grupal. 
Esta aseveración coincide con la opinión emitida por Velásquez y López (2008) quienes plantean queen la gestión escolar y administrativa, se ofrecen programas tecnológicos, profesionales y cursos de posgrado sin ningún tipo de diagnóstico o respaldo en investigaciones que consulten la realidad del contexto. Se compran equipos, software, se adquieren costosas bases de datos y se contrata personal, sin haber hecho lectura de la realidad sustentada en investigaciones. El uso de las TIC debe considerar instrumentos de integración de contenidos, así como herramientas para el proceso de enseñanza aprendizaje, generando situaciones susceptibles de ser sometidas al análisis, evaluación e investigación permanente (Enrique y Alzugaray, 2013). En virtud de lo antes expuesto, esta investigación se propuso como objetivo analizar dos de las dimensiones del empoderamiento digital, relacionadas con la equidad digital y la empleabilidad dentro del sector universitario colombiano, específicamente en la Universidad de La Guajira (UniGuajira), con el fin de proponer reorientaciones curriculares que contribuyan a fomentar su desarrollo.

Dicha universidad está conformada por cinco facultades, a saber, Ciencias Básicas, Educación, Ingeniería, Ciencias Económicas y Ciencias Sociales. La planta profesoral que atiende a estas facultades se clasifica según el tiempo de vinculación con la universidad y de acuerdo con el nivel de formación académica. Con respecto al tiempo de dedicación a la universidad se tienen tres estratos; docentes a tiempo completo o de planta, ocasionales y catedráticos, siendo estos últimos los que tienen menores compromisos laborales dada su condición de contratados. La preparación académica de estos docentes varía desde profesionales, especialistas, doctores y postdoctores.

\section{METODOLOGÍA}

Para el desarrollo de este trabajo se empleó un enfoque cuali-cuantitativo. El aspecto cualitativo se manifiesta a través de su diseño el cual se corresponde con un estudio de caso, definido por Quaranta (2006) como aquel que recurre a diseños metodológicos que pueden combinar procedimientos cualitativos y cuantitativos y que tiene por objetivo el estudio de un sistema, delimitado en tiempo y espacio de actores, relaciones e instituciones sociales. En este sentido, el estudio se delimitó al espacio correspondiente al Departamento de La Guajira, tomando en consideración actores o representantes de cada uno de los estratos que conforman la planta docente de La Universidad de La Guajira durante el año lectivo de 2016.

En cuanto al enfoque cuantitativo, en este estudio se utiliza una metodología de investigación descriptiva, no experimental, transeccional y de campo (Hernández et al., 2011). Dicha metodología se evidencia a través del conteo de los datos arrojados por la muestra para cuyo análisis se recurrió a la estadística descriptiva, mediante el uso de histogramas de frecuencias que ilustran el comportamiento de la media aritmética, como medida de tendencia central. En primer lugar, se revisaron los documentos relacionados con las dimensiones del estudio, los cuales sirvieron de referentes teórico-conceptuales para describir y analizar las respuestas de la muestra. Estas respuestas se obtuvieron en el mismo campo donde se realizó la investigación, es decir, en las diferentes facultades de UniGuajira, en un solo momento, lo que la caracteriza como transeccional.

\section{Procedimiento para la recolección de información}

A partir de la revisión documental realizada se procedió al diseño del instrumento de recolección de datos, tipo cuestionario el cual fue sometido a las pruebas de validez y confiabilidad pertinentes, arrojando como resultado 0,87 , lo cual convierte al cuestionario en un instrumento confiable. El mismo constó de dos partes que sirvieron para medir tanto los aspectos cualitativos como los cuantitativos. En la primera parte se indagaron los datos personales de la muestra, tales como formación académica, tiempo de vinculación con la universidad y facultad a la cual pertenece. En la segunda parte, se incluyeron 10 preguntas, 5 de ellas relacionadas con la equidad digital y el resto con la gestión de empleo. Dicho cuestionario contó con una escala de frecuencia de 5 opciones de respuestas la cual varió desde: muy alta con escala de 5 , alta con escala de 4, moderado con 3, baja con 2, hasta muy baja con 1 . Esta escala sirvió para medir tanto el grado de formación universitaria basado en la equidad, como la formación para la empleabilidad; dimensiones éstas derivadas del empoderamiento digital. Los intervalos utilizados para calcular la media de los resultados se presenta en la Tabla 2.

Tabla 2: Intervalos para el cálculo de la media.

\begin{tabular}{|c|c|l|l|}
\hline Valor & Intervalos & Alternativas & Categoría \\
\hline 5 & $4,21-5.0$ & Se cumple plenamente & Muy Alto \\
\hline 4 & $3,41-4,2$ & Se cumple en alto grado & Alto \\
\hline 3 & $2,61-3,4$ & Se cumple aceptablemente & Moderado \\
\hline 2 & $1,81-2,6$ & Se cumple insatisfactoriamente & Bajo \\
\hline 1 & $1-1,8$ & No se cumple & Muy Bajo \\
\hline
\end{tabular}




\section{Población y muestra}

La población de esta investigación estuvo constituida por los tres estratos que conforman el cuerpo profesoral de la UniGuajira, de acuerdo con su vinculación a la universidad, a saber: 170 docentes de planta, 219 docentes ocasionales y 869 catedráticos, los cuales suman un total de 1.258. Para garantizar la calidad de los datos, el muestreo fue de tipo no probabilístico, tomando una muestra (n) aleatoria representativa del $10 \%$, equivalente a 125 profesores, tal como se detalla en la Tabla 3. Esta selección muestral se sustenta en los planteamientos de Ary et al. (2008) quienes establecen que para las investigaciones descriptivas basta con seleccionar de un 10 a un $20 \%$ de la población sujeta a estudio.

Tabla 3: Procedimiento para la selección muestral por estratos.

\begin{tabular}{|c|c|c|}
\hline Estratos (vinculación con la universidad) & Población & Muestra (10\%) \\
\hline Docentes de planta & 170 & 17 \\
\hline Docentes ocasionales & 219 & 21 \\
\hline Catedráticos & 869 & 87 \\
\hline Total & 1.258 & 125 \\
\hline
\end{tabular}

Vale acotar que este muestreo se realizó de manera estratégica, fijando como criterios de inclusión: representantes de cada uno de los estratos, con conocimientos sobre el manejo de las TIC en educación superior y voluntad para el suministro de los datos (Hernández et al., 2011). Los aspectos cualitativos de estos representantes de la muestra, tales como nivel de formación académica y pertenencia a las diferentes facultades fueron indagados mediante el instrumento de recolección de datos para así verificar su participación proporcional dentro de la población.

\section{Procedimiento para el análisis de los datos}

El análisis cualitativo de los datos requirió agrupar los cuestionarios según los estratos, el nivel de formación académica y la pertenencia de la muestra en las diferentes facultades de la UniGuajira. A tal efecto, se utilizó una matriz o tabla de registro que facilitó distribuir la muestra seleccionada de acuerdo con los criterios mencionados. Después de obtener la información cualitativa, se construyó una base de datos en Excel 2013 para WINDOWS, en el cual se introdujeron las respuestas dadas por la muestra. Los datos fueron graficados utilizando histogramas de frecuencias relativas, correspondientes al tipo de metodología descriptiva, para su mejor análisis.

\section{RESULTADOS Y DISCUSIÓN}

Este apartado de presentación de los resultados con su respectivo análisis ha sido dividido en tres secciones, atendiendo al carácter cuali-cuantitativo de la investigación. En la primera, se reportan las características cualitativas de la muestra las cuales fueron extraídas de la primera parte del Cuestionario. Las secciones restantes están relacionadas con el análisis cuantitativo de las dos dimensiones del empoderamiento digital sometidas a evaluación a saber, equidad digital y empleabilidad.

Características cualitativas de la muestra:

Tal como se mencionó en los párrafos precedentes, la primera parte del Cuestionario se limitó a indagar información sobre los datos personales de la muestra, los cuales se reportan en la tablas 4 y 5.

Tabla 4: Resultados de la distribución de la muestra por facultades

\begin{tabular}{|l|c|c|c|c|c|c|}
\hline \multirow{2}{*}{ Estratos } & \multicolumn{5}{|c|}{ Facltadess } & Total \\
\cline { 2 - 8 } & Ciencias Básicas & Educación & Ingeniería & Ciencias Económicas & $\begin{array}{l}\text { Ciencias } \\
\text { Sociales }\end{array}$ & \\
\hline Docentes de planta & 4 & 3 & 4 & 3 & 3 & 17 \\
\hline Docentes ocasionales & 8 & 7 & 3 & 2 & 25 & 87 \\
\hline Catedráticos & 25 & 12 & 10 & 15 & Total (N) & 125 \\
\hline
\end{tabular}

Tabla 5: Resultados de la distribución de la muestra según formación académica

\begin{tabular}{|l|c|c|c|c|c|c|}
\hline \multirow{2}{*}{ Estratos } & \multicolumn{5}{|c|}{ FormaciónAcadémica } & \multirow{2}{*}{ Total } \\
\cline { 2 - 6 } & Profesionales & Especialistas & Magister & Doctores & Postdoctores & \\
\hline Docentes de planta & & & 13 & 4 & & 17 \\
\hline Docentes ocasionales & & 6 & 14 & 1 & & 21 \\
\hline Catedráticos & 50 & 20 & 17 & & & 87 \\
\hline
\end{tabular}


Los resultados arrojados mediante el proceso de agrupación de los cuestionarios, desde el punto de vista cualitativo, demuestran una distribución heterogénea, tanto en lo que respecta a la ubicación de la muestra en las cinco facultades como en su formación académica. Esta ubicación y caracterización sirvió para sustentar los resultados cuantitativos arrojados por esta selección muestral en relación con la equidad y empleabilidad, como dimensiones del empoderamiento digital que seguidamente se discuten y analizan. Equidad digital

La Tabla 6 presenta los resultados en frecuencias absolutas correspondientes a la equidad o igualdad digital mientras que, la Tabla 7 muestra dichos resultados en frecuencias relativas las cuales son ilustradas en la Figura 2.

Tabla 6: Distribución de frecuencias absolutas para la dimensión equidad digital, suministradas por la muestra.

\begin{tabular}{|c|c|c|c|c|c|c|}
\hline \multirow{2}{*}{ Dimensión } & \multirow{2}{*}{ Preguntas } & \multicolumn{5}{|c|}{ Respuestas $(n=125)$} \\
\hline & & 1 & 2 & 3 & 4 & 5 \\
\hline \multirow{5}{*}{$\begin{array}{c}\text { Equidad o } \\
\text { igualdad digital }\end{array}$} & $\begin{array}{l}\text { 1. ¿La educación universitaria en Colombia dota de habilidades digitales a } \\
\text { sus profesores en términos de equidad e igualdad? }\end{array}$ & 0 & 30 & 70 & 25 & 0 \\
\hline & $\begin{array}{l}\text { 2. ¿La educación universitaria en Colombia dota de habilidades digitales a } \\
\text { sus alumnos en términos de equidad e igualdad? }\end{array}$ & 0 & 25 & 80 & 20 & 0 \\
\hline & $\begin{array}{l}\text { 3. ¿Para la implementación de programas tecnológicos se realiza un } \\
\text { estudio diagnóstico destinado al análisis de la realidad del contexto } \\
\text { estudiantil? }\end{array}$ & 0 & 0 & 100 & 25 & 0 \\
\hline & $\begin{array}{l}\text { 4. ¿La implementación de programas tecnológicos se basa en } \\
\text { investigaciones que consulten la realidad del contexto en donde se } \\
\text { desenvuelve el docente? }\end{array}$ & 0 & 0 & 90 & 35 & 0 \\
\hline & $\begin{array}{l}\text { 5. ¿Los programas tecnológicos implementados por las universidades } \\
\text { colombianas, desarrollan y promueven procesos de mejora de las } \\
\text { condiciones de vida, comunicación, participación y poder de los grupos } \\
\text { menos atendidos? }\end{array}$ & 0 & 10 & 95 & 20 & 0 \\
\hline
\end{tabular}

Tabla 7: Distribución de frecuencias absolutas $(\mathrm{Fa})$ y relativas $(\mathrm{Fr})$ sobre la dimensión equidad digital.

\begin{tabular}{|c|c|c|c|c|c|c|c|c|c|c|}
\hline Pregunta & \multicolumn{2}{|c|}{1} & \multicolumn{2}{|c|}{2} & \multicolumn{2}{|c|}{3} & \multicolumn{2}{|c|}{4} & \multicolumn{2}{|c|}{5} \\
\hline Respuesta & $\mathrm{Fa}$ & Fr\% & $\mathrm{Fa}$ & $\mathrm{Fr} \%$ & $\mathrm{Fa}$ & Fr\% & $\mathrm{Fa}$ & $\mathrm{Fr} \%$ & $\mathrm{Fa}$ & $\mathrm{Fr} \%$ \\
\hline 1 & 0 & 0 & 0 & 0 & 0 & 0 & 0 & 0 & 0 & 0 \\
\hline 2 & 30 & 24 & 25 & 20 & 0 & 0 & 0 & 0 & 10 & 8 \\
\hline 3 & 70 & 56 & 80 & 64 & 100 & 80 & 90 & 72 & 95 & 76 \\
\hline 4 & 25 & 20 & 20 & 16 & 25 & 20 & 35 & 28 & 20 & 16 \\
\hline 5 & 0 & 0 & 0 & 0 & $v$ & 0 & 0 & 0 & 0 & 0 \\
\hline Sumatoria & 125 & 100 & 125 & 100 & 125 & 100 & 125 & 100 & 125 & 100 \\
\hline Media & \multicolumn{2}{|c|}{2,9} & \multicolumn{2}{|c|}{2.96} & \multicolumn{2}{|c|}{3,20} & \multicolumn{2}{|c|}{3,28} & \multicolumn{2}{|c|}{3.08} \\
\hline
\end{tabular}

Desarrollo de procesos para grupos menos atendidos

Investigaciones para estudiar la realidad del contexto

Diagnóstico para estudiar la realidad del contexto

Habilidades digitales con equidad para alumnos

Hab. Digitales con equidad para profesores

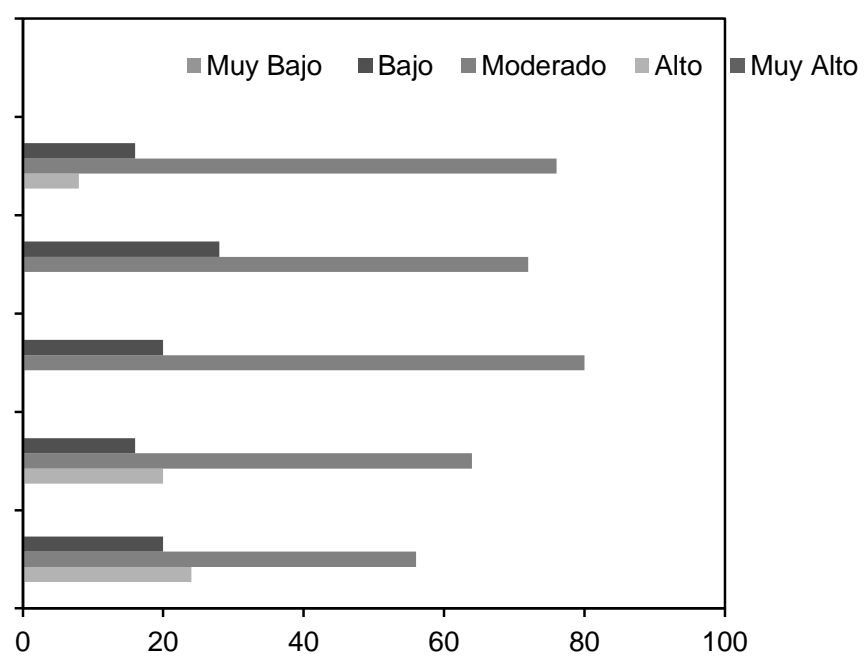

Fig.2: Distribución porcentual de los resultados sobre equidad digital 
Puede observarse en la Tabla 7 que la media resultante se ubicó en 3,28 lo cual posiciona la formación universitaria que ofrece UniGuajira, en términos de equidad digital, en la categoría de moderado. Esta ubicación en la mitad de la frecuencia escalar demuestra un claro rezago en las posibilidades de acceso en condiciones de equidad a las TIC tanto para los profesores quienes reportaron un $56 \%$ en el rango de moderado, como para los estudiantes que se encuentran en formación dentro de este contexto, manifestándose en esta misma categoría en un $64 \%$. Se infiere, entonces, que los planteamientos hechos por Tedesco (2005) y que atañen al sentido de equidad digital en Latinoamérica aún están pendientes por superarse dentro de este contexto universitario colombiano.

Quizás, factores tales como la segregación escolar de los grupos desaventajados y vulnerables; el desfase tecnológico, metodológico y didáctico imperante en las instituciones de formación pública; el desgranamiento de los contenidos curriculares; la inadecuada formación de los profesores y la dispersión de las inversiones financieras en el campo educativo y especialmente en el universitario; mencionados por Tedesco (2005), no han encontrado una total solución en esta región de Latinoamérica. En relación con éste último aspecto, también hay que considerar la opinión sustentada por Velásquez y López (2008) en lo que se refiere a que los programas implementados para lograr la equidad digital no estén dando respuestas a las necesidades educativas de la región. Así lo evidencia un $80 \%$ y $72 \%$ de la muestra encuestada en los ítems 4 y 5 respectivamente cuando se refieren a la falta de diagnóstico o respaldo en investigaciones que consulten la realidad del contexto. El estado moderado, resultante en esta dimensión también indica que se debe trabajar en la eliminación de las brechas tecnológicas que puedan llegar a discriminar algunos sectores en este mundo permeado por las TIC (Bustos y Coll, 2010), así como también en acreditar al estudiante con el desarrollo de competencias que son potencialmente útiles en las actividades profesionales y a la sociedad en general (Coll y Zegwaard, 2006).

Desde el punto de vista cualitativo, vale acotar que los porcentajes obtenidos en lo que respecta al ítem 1 , fueron reportados por docentes catedráticos, provenientes de las Facultades de Ciencias Básicas y Ciencias Sociales, cuyos niveles de formación se corresponden con los de profesionales y especialistas. Esta ubicación porcentual pudiera estar relacionada con el tiempo de dedicación que tienen estos docentes con la universidad así como también su nivel de formación. De allí que su opinión con respecto a la dotación de habilidades digitales por parte de la UniGuajira en términos de equidad se orientará hacia el nivel moderado.

\section{Empleabilidad}

Al igual que en la dimensión anterior, los resultados en frecuencias absolutas, arrojados por la muestra, de 125 docentes sobre la gestión de empleo se reportan en la Tabla 8, las relativas en la Tabla 9 y su ilustración en la Figura 3.

Tabla 8: Distribución de frecuencias absolutas para la dimensión gestión de empleo, suministradas por la muestra.

\begin{tabular}{|l|l|l|l|l|l|l|}
\hline Dimensión & Preguntas & \multicolumn{3}{|c|}{ Respuestas $(\mathrm{n}=125)$} \\
\hline & $\begin{array}{l}\text { 6. ¿La educación universitaria en Colombia es susceptible de reorientar la } \\
\text { formación profesional relacionada con las TIC para facilitar la empleabilidad? }\end{array}$ & 0 & 15 & 98 & 12 & 0 \\
\cline { 2 - 7 } & $\begin{array}{l}\text { 7. ¿La programación de los contenidos curriculares en las universidades } \\
\text { colombianas incluyen metodologías actualizadas derivadas de la TIC? }\end{array}$ & 0 & 0 & 100 & 25 & 0 \\
\hline & $\begin{array}{l}\text { 8. ¿Los programas de preparación universitaria colombiana están vinculados } \\
\text { Eon las exigencias del mercado laboral en cuanto al uso de las TIC? }\end{array}$ & 0 & 0 & 90 & 35 & 0 \\
\hline & $\begin{array}{l}\text { 9. ¿La educación universitaria colombiana incluye el desarrollo de } \\
\text { Empetencias tecnológicas adecuadas para que los egresados puedan } \\
\text { insertarse en el mercado laboral? }\end{array}$ & 0 & 10 & 95 & 20 & 0 \\
\cline { 2 - 7 } & $\begin{array}{l}\text { 10 ¿La formación profesional relacionada con las TIC es evaluada de manera } \\
\text { continua en términos de adaptación a las necesidades del mundo laboral? }\end{array}$ & 0 & 0 & 98 & 27 & 0 \\
\hline
\end{tabular}

Tabla 9: Distribución de frecuencias absolutas (Fa) y relativas (Fr) sobre la dimensión gestión de empleo.

\begin{tabular}{|l|c|c|c|c|c|c|c|c|c|c|}
\hline Pregunta & \multicolumn{2}{|c|}{6} & \multicolumn{2}{|c|}{7} & \multicolumn{2}{|c|}{8} & \multicolumn{2}{|c|}{9} & \multicolumn{2}{c|}{10} \\
\hline Respuesta & Fa & Fr\% & Fa & Fr\% & Fa & Fr\% & Fa & Fr\% & Fa & Fr\% \\
\hline 1 & 0 & 0 & 0 & 0 & 0 & 0 & 0 & 0 & 0 & 0 \\
\hline 2 & 15 & 12 & 0 & 0 & 0 & 0 & 10 & 8 & 0 & 0 \\
\hline 3 & 98 & 78,4 & 100 & 80 & 90 & 72 & 95 & 76 & 98 & 78,4 \\
\hline 4 & 12 & 9,6 & 25 & 20 & 35 & 28 & 20 & 16 & 27 & 21,6 \\
\hline 5 & 0 & 0 & 0 & 0 & 0 & 0 & 0 & 0 & 0 & 0 \\
\hline Sumatoria & 125 & 100 & 125 & 100 & 125 & 100 & 125 & 100 & 125 & 100 \\
\hline Media & 2,98 & \multicolumn{1}{|c|}{3,20} & 3,28 & 3,08 & 3,22 \\
\hline
\end{tabular}


Como puede observarse en Tabla 9, la media resultante de la dimensión formación universitaria para la empleabilidad se ubicó en 3,28, posicionándose, de forma similar a la dimensión anterior, en la categoría moderado. Llama la atención los resultados arrojados por la preparación universitaria en torno a la empleabilidad, en los cuales se esperaba la máxima calificación, dada la vigilancia permanente que mantienen los Ministerios de Educación y Tecnología y los repertorios legales que garantizan su ejecución presentados en la Tabla 1. Estos resultados invitan a revisar la gestión asignada por el MinTIC al Observatorio Laboral de Educación el cual tiene en sus manos la labor de velar por: la pertinencia de la formación adquirida por el estudiante en este nivel educativo con relación a las TIC, el vínculo con el mercado laboral y otras variables que inciden en la empleabilidad. Así mismo, es necesario reconsiderar lo planteado por Martínez et al. (2015) en cuanto a la responsabilidad del sector universitario de empoderar digitalmente al individuo en formación para que sea capaz de insertarse en el mundo laboral con las competencias que debe acreditar (Coll y Zegwaard, 2006) las cuales le permitirán enlazar su formación con las necesidades del mercado.

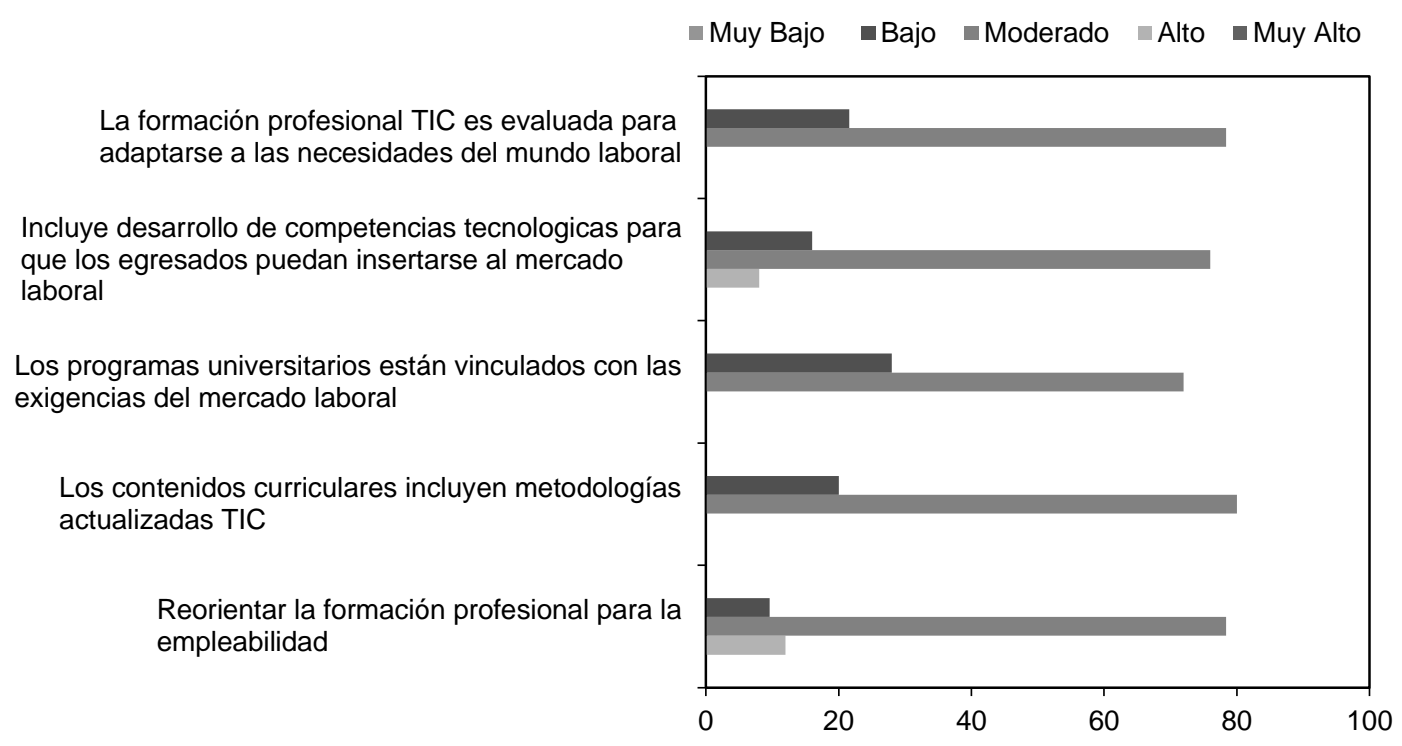

Fig.3: Distribución porcentual de los resultados sobre empleabilidad

En términos cualitativos, vale la pena mencionar que de las 98 respuestas ubicadas en el ítem 1, relacionadas con la reorientación de la formación profesional para el uso de las TIC y así facilitar la empleabilidad, 87 de las mismas fueron reportadas por los docentes clasificados en la categoría de catedráticos. Al igual que en la dimensión anterior estas respuestas conducen a inferir su poco interés en darle prioridad a las modificaciones curriculares orientadas al uso de las TIC que hacen más factible la inserción del futuro profesional en el mercado laboral, tal como lo plantean Belloso y Perozo (2009).

En resumen, puede afirmarse que los valores reportados por la muestra no dejan de ser preocupantes para el contexto colombiano, específicamente para la UniGuajira, al catalogarse en ambas dimensiones de empoderamiento digital en la categoría moderado. Esto conduce a pensar que los acelerados cambios exigidos por la implementación de las TIC no se han aplicado de manera inmediata, sobre todo por tratarse de una estructura organizativa tan compleja como lo es la universidad, en la que la implementación de procedimientos y metodologías dependen de la gestión de la enorme cantidad de actores participantes, de una adecuada infraestructura tecnológica que permita establecer redes de comunicación colaborativa para definir metas digitales, analizar tendencias y evaluar efectos e impactos.

\section{DISCUSIÓN FINAL}

En función de los resultados presentados en el apartado anterior, a continuación, se sugieren algunas reorientaciones curriculares que pudieran tomarse en cuenta para elevar el nivel alcanzado por la UniGuajira, hacia la máxima categoría. Tales reorientaciones se basan en los referentes teóricoconceptuales arriba descritos y tenderán a optimizar las prácticas educativas en este entorno, específicamente aquellas relacionadas con la equidad digital y la empleabilidad como dimensiones del empoderamiento digital. A continuación, se describen estas reorientaciones

Entre las prácticas que requieren verdaderos cambios en lo interno a nivel universitario, se encuentran las dinámicas pedagógicas relacionadas con la planificación y el diseño didáctico en términos de equidad digital. Una aplicación significativa de las tecnologías digitales con miras a un mayor desarrollo, promoción y 
empoderamiento, requiere algo más que la introducción esporádica de una herramienta. Exige una revisión y cambio desde el diseño de la enseñanza, la renovación metodológica y el compromiso de la vigilancia epistemológica y permanente de los procesos didácticos generados por todos y para todos, en la imbricada interacción entre el plan minucioso, la práctica y sus emergentes. Cada universidad debe responder desde su propia especificidad, partiendo del contexto en el que se halle, considerando la sociedad a la que debe servir, teniendo en cuenta la tradición y las fortalezas que posee.

Para los profesores, es necesario la creación de un sistema de apoyo que incluya tanto las acciones del plan de formación y actualización del profesorado respecto al uso de las TIC en la docencia, como todo el sistema de asesoría personal que se presta a los mismos, con respecto a las acciones de asistencia técnica. Estos sistemas de apoyo no sólo deben centrarse en el papel fundamental de la formación del profesorado sino, además, en el ejercicio de formación continua para su desarrollo profesional. Esto le permitiría entre otras cosas, estar al día con los descubrimientos en su campo de estudio, atender a las posibilidades que les ofrece las TIC, procesar las eventuales innovaciones en los procesos de enseñanzaaprendizaje y diseñar estrategias que satisfagan las necesidades de sus estudiantes de acuerdo con las diversidades personales, sociales, culturales y étnicas.

Los alumnos también requieren acciones de formación, tales como destrezas comunicativas, de selección de la información, de organización, entre otras, pero, al mismo tiempo, asistencia técnica y políticas de promoción del uso de las TIC. La voluntad política e inclusión del empoderamiento digital en los distintos planes de desarrollo de la región no son garantía de su éxito. Se necesita también el ejercicio permanente de identificación de aquellas variables que estén afectando el normal desarrollo del proceso académico y de adaptación a la vida universitaria para intervenirlas de manera preventiva en la mayoría de los casos, y correctiva cuando no se tenga otra opción. Esto exige una evaluación continua de dichos planes, sobre todo, si no se adaptan a las necesidades reales del contexto.

Dentro de estas reorientaciones curriculares hay que considerar que las modalidades de formación apoyadas en las TIC llevan a nuevas concepciones del proceso de enseñanza-aprendizaje que acentúan la implicación activa del alumno en el proceso de aprendizaje; la atención a las destrezas emocionales e intelectuales a distintos niveles; la preparación de los jóvenes para asumir responsabilidades en un mundo en rápido y constante cambio; la flexibilidad de los alumnos para entrar en un mundo laboral que demandará formación a lo largo de toda la vida; y las competencias necesarias para involucrarse en este proceso de aprendizaje continuo.

Finalmente, vale acotar que las reorientaciones curriculares sugeridas no constituyen una panacea sobre los resultados encontrados. Es importante seguir avanzando en capacitación docente para el uso pedagógico de las TIC, ofrecer apoyo a los estudiantes para su verdadero empoderamiento digital y crear una cultura digital en la cual las interacciones universitarias funcionen como círculos virtuosos acordes con el mundo globalizado.

\section{CONCLUSIONES}

Las conclusiones derivadas de esta investigación se basan en el objetivo que se propuso alcanzar a través de la misma. Éste consistió en analizar la equidad y la empleabilidad, como dos de las dimensiones del empoderamiento digital en el contexto universitario colombiano a fin de proponer reorientaciones curriculares que contribuyan a fomentar su desarrollo.

Con respecto a la equidad dentro del sector universitario, que para esta investigación se asumió como la dotación de habilidades digitales a profesores y alumnos con sentido de igualdad, se pudo observar que Colombia no sólo se ha preocupado por esta dotación, sino que la misma se encuentra consagrada en repertorios reglamentarios y legales que garantizan su puesta en marcha. A pesar de que los resultados ubican esta dotación en el nivel moderado, pudiera ocurrir que la misma se encuentra en constante movimiento, dado los acelerados cambios suscitados por las innovaciones producidas en el campo de las TIC. En todo caso, se sugiere para la comunidad académica universitaria, sistemas de apoyo técnico que logren ponerla al tanto de las revoluciones tecnológicas que se suscitan en la actual sociedad del conocimiento.

En relación con la preparación digital para la empleabilidad, no cabe duda de los esfuerzos que realiza este país para vigilar la formación del futuro profesional acorde con las necesidades tecnológicas del entorno. Sin embargo, tales esfuerzos no pueden darse por terminados pues es imprescindible considerar la demanda social que exige profesionales cada vez más especializados. Ello en virtud de la creciente innovación tecnológica que, tanto en procesos de producción de bienes como de gestión de servicios, se está produciendo gracias a las TIC. 


\section{REFERENCIAS}

Ary, D. y otros tres autores ( $5^{\mathrm{a}}$ Ed.), Introducción a la Investigación Pedagógica. Interamericana: México (2008)

Bauman, Z. (15 ${ }^{\mathrm{a}}$ Ed.), Dentro della globalizzazione. Le conseguenze sulle persone, 25-30. Laterza: Bari, Roma (2005)

Berrío-Zapata, C. y H. Rojas, La brecha digital universitaria: La apropiación de las TIC en estudiantes de educación superior en Bogotá (Colombia), Revista Comunicar: 43(22), 133-142 (2014)

Berrío-Zapata, C., Una visión crítica de la intervención en tecnologías de la información y comunicación (TIC) para atacar la brecha digital y generar desarrollo sostenible en comunidades carenciadas en Colombia: el Proyecto Cumaribo, Management: XIV (23-24), 165-181 (2005)

Bustos, A. y C. Coll, Los entornos virtuales como espacios de enseñanza y aprendizaje. Una perspectiva psicoeducativa para su caracterización y análisis, Rev. Mexicana de Invest. en Educación: 15(44), 163-184 (2010)

Catts, R. y J. Lau, Towards information Literacy Indicators. UNESCO: Paris (2008)

Coll, R.K. y K. Zegwaard, Perceptions of desirable graduate competencies for science and technology new Graduates, Research in Science \& Technological Education: 24 (1), 29-58 (2006)

De La Fontaine, D. y P.C. Aparicio ( $1^{\mathrm{a}}$ Ed.), Diversidad cultural y desigualdad social en América Latina y el Caribe desafíos de la integración global, 155-198. Heinrich Böl: San Salvador, El Salvador (2008)

Enrique, C. y G. Alzugaray, Modelo de Enseñanza-Aprendizaje para el Estudio de la Cinemática de un Volante Inercial usando Tecnologías de la Información y la Comunicación en un Laboratorio de Física, Revista Formación Universitaria: 6(1), 3-12 (2013)

Galvis, A. ( $1^{\text {a } E d .), ~ L a s ~ p o l i ́ t i c a s ~ T I C ~ e n ~ l o s ~ s i s t e m a s ~ e d u c a t i v o s ~ d e ~ A m e ́ r i c a ~ L a t i n a: ~ C a s o ~ C o l o m b i a, ~ 94-95 . ~}$ UNICEF: Buenos Aires, Argentina (2014)

Gisbert, M. y F. Esteve, Digital learners: La competencia digital de los estudiantes universitarios, Revista La Cuestión Universitaria: 7, 48-59 (2011)

Granados-Romero, J. y otros cinco autores, Las tecnologías de la información y las comunicaciones, las del aprendizaje y del conocimiento y las tecnologías para el empoderamiento y la participación como instrumentos de apoyo al docente de la universidad del siglo XXI, Medisur: 12(1) (2014)

Hernández, R., C. Fernández y P. Baptista ( $5^{\text {ta }}$ Ed.), Metodología de la investigación, 612-613. McGraw-Hill Interamericana: Ciudad de México, México (2011)

Martínez, E. y otros tres autores, An application of the performance-evaluation model for e-learning quality in higher education, Total Quality Management \& Business Excellence: 26(5-6), 632-647 (2015)

Mejía, J. y D. López, Modelo de Calidad de E-learning para Instituciones de Educación Superior en Colombia, Revista Formación Universitaria: 9(2), 59-72 (2016)

MinTIC (Ministerio de Tecnología de Información y Comunicación), Plan Nacional de Tecnologías de la Información y las Comunicaciones 2008-2019 (https://goo.gl/DTPHqL, acceso: julio 17 2016) (2008)

OEI (Organización de Estados Iberoamericanos para la Educación, la Ciencia y la cultura). Metas Educativas 2021, La educación que queremos para la generación de los bicentenarios. OEI: Madrid (2010)

Quaranta, G., "Los estudios de caso en la investigación sociológica". En: de Gialdino, Vasilachis (comp.), Estrategias de investigación cualitativa. Gedisa: Buenos Aires (2006)

Quintero, J., D. Parra y D. Araujo, Integración de las tecnologías de información y comunicación (TIC) en la práctica docente en la Universidad de la Guajira, Revista Telematique: 10(1),17-37 (2011)

Tedesco, J.C., Tecnologías de la Información y desigualdad educativa en América Latina (en la web: https://goo.gl/rWe9qy, acceso: julio 15 de 2016) (2005)

Velásquez, A. y E. López, Una mirada crítica al papel de las TIC en la educación superior en Colombia, Revista E-mail Educativo: 1(1), 1-9 (2008)

Venkatesh, V. y Sykes, T., Digital Divide Initiative Success in Developing Countries: A Longitudinal Field Study in a Village in India, Information Systems Research: 24(2): 239-260 (2013)

WEB Foundation, Informe publicado en el Diario en línea El Espectador, Sección Tecnología (en la web: https://goo.gl/EdktwD, acceso: 13 de abril 2016), (2015) 
